\title{
Anharmonic potential functions as derived from Raman intensities: Methane
}

Cite as: J. Chem. Phys. 81, 3835 (1984); https://doi.org/10.1063/1.448166

Submitted: 30 November 1983 . Accepted: 19 June 1984 . Published Online: 31 August 1998

D. Bermejo, and S. Montero

\section{ARTICLES YOU MAY BE INTERESTED IN}

Calculation of converged rovibrational energies and partition function for methane using vibrational-rotational configuration interaction

The Journal of Chemical Physics 121, 2071 (2004); https://doi.org/10.1063/1.1759627

Vibrational zero-point energies and thermodynamic functions beyond the harmonic approximation

The Journal of Chemical Physics 120, 3059 (2004); https://doi.org/10.1063/1.1637580

Vibrational Energy Transfer in Methane

The Journal of Chemical Physics 49, 1111 (1968); https://doi.org/10.1063/1.1670199
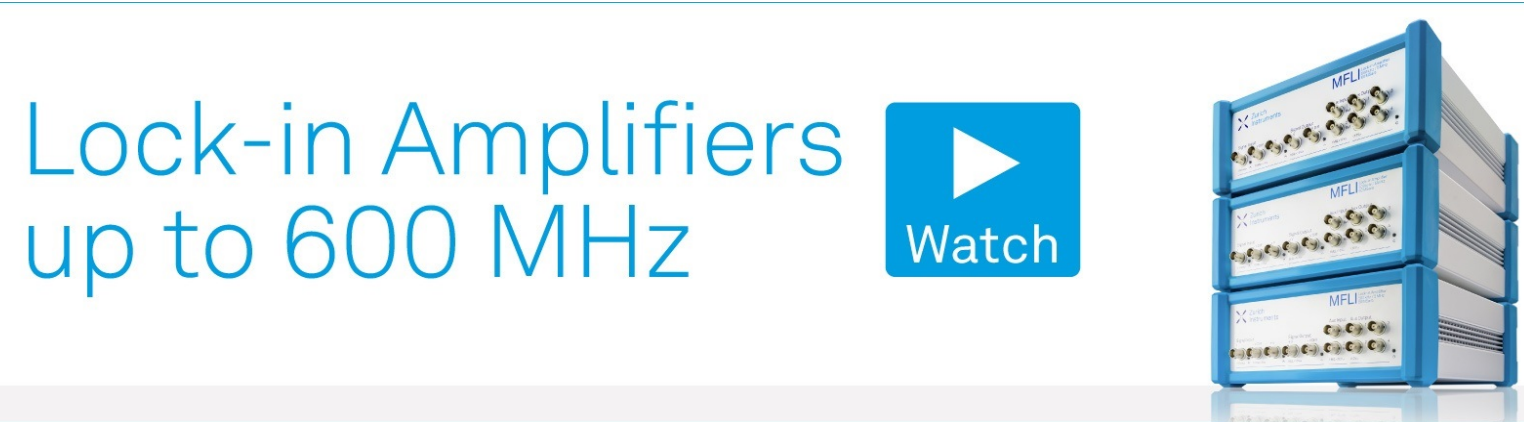


\title{
Anharmonic potential functions as derived from Raman intensities: Methane ${ }^{a)}$
}

\author{
D. Bermejo and S. Montero \\ Instituto de Estructura de la Materia, CSIC, Serrano 119, Madrid (6), Spain
}

(Received 30 November 1983; accepted 19 June 1984)

The cubic force field of methane has been investigated on the basis of the analysis of Raman scattering cross sections of binary overtones and combination bands. Seven absolute cross sections, ten symmetry cubic force constants, and the second derivative of the mean $\mathrm{CH}$ bond polarizability are the original results of present work. Some of these cubic force constants are determined with high accuracy. Most $\widehat{H}_{22}$ rovibrational constants calculated by means of present anharmonic force field are in good agreement with the experimental values reported by various authors.

\section{INTRODUCTION}

Deriving the anharmonic vibrational potential function of polyatomic molecules is one of the most difficult problems of present day spectroscopic research. For most small polyatomic molecules, attempts have been made to establish the first cofficients of the potential function, namely, the quadratic, cubic, and quartic force constants. The problem is so complex that only in very few cases, $\mathrm{CO}_{2}$ for instance, ${ }^{1}$ these force constants are firmly established.

In the case of methane, the cubic force field has been investigated by various authors, ${ }^{2-8}$ but the lack of agreement is considerable for most cubic force constants. Besides that, for the quadratic force field still are doubts arising from the $v_{2}: v_{4}$ Coriolis resonances in $\mathrm{CH}_{4}$ and $\mathrm{CD}_{4}$ and $v_{2}+v_{4}: v_{1}$ in $\mathrm{CD}_{4}$. About the quartic force constants almost nothing is known.

With few exceptions, ${ }^{9}$ the force field has been hitherto investigated according to two different procedures, a theoretical one, based on $a b$ initio calculations, and an empirical one based on the analysis of the experimental wave numbers of the rotation-vibration bands, in connection with an approximate expression for the Hamiltonian operator. For methane, the first method was used in Refs. 2-4 and the second in Refs. 6 and 7.

A different approach has been followed in present work, since the main source of information has been the experimental Raman scattering cross sections of first and second order vibrational spectrum, using the bond polarizability model in connection with the anharmonic treatment of Raman intensities to the cubic term. With little theoretical effort, the method might be extended to the quartic term, but the necessary experimental intensity data are not yet available.

The method here proposed seems to be quite efficient, in so far as the Raman cross sections of overtones and combination bands are extremely sensitive to the vibrational interactions (mainly Fermi resonances) governed by cubic force constants. For these bands, changes of up to three orders of magnitude with respect to the calculated unperturbed cross section are observed. In such cases, the cross section of the Fermi diad $v_{a}: 2 v_{b}$ or $v_{a}: v_{b}+v_{c}$ yields highly localized information on the cubic force constant $k_{a b b}$ or $k_{a b c}$, respec-

a) Issued as CSIC No. FM49 tively.

Ten cubic force constants in symmetry coordinates $F_{i j k}$ common to all isotopic derivatives of methane have been derived here by using the absolute cross sections of 14 overtone or combinations bands of the species ${ }^{12} \mathrm{CH}_{4},{ }^{12} \mathrm{CD}_{4}$, ${ }^{12} \mathrm{CH}_{3} \mathrm{D}$, and ${ }^{12} \mathrm{CH}_{2} \mathrm{D}_{2}$. Since the possible number of useful intensity data on methane isotopic species is well over 200 , the possibilities of the present method for establishing the complete set of 13 symmetry cubic constants $F_{i j k}$ are still very large.

As an intermediate result of present work, all second derivatives of the mean molecular polarizability of methane species with respect to symmetry and normal coordinates were obtained. They may be useful for further refinement of the cubic force field by including new intensity data. Those of ${ }^{12} \mathrm{CH}_{4},{ }^{13} \mathrm{CH}_{4},{ }^{12} \mathrm{CD}_{4}$, and ${ }^{13} \mathrm{CD}_{4}$ are reported in Table II.

\section{EXPERIMENTAL}

Commercial samples of methane deuterated derivatives (Stohler, Isotopical Center) were used for recording most spectra. For $\mathrm{CD}_{4}$, a new sample was prepared by us, in order to verify some features in the $v_{3}+v_{4}$ band, which in a preliminary analysis of the data were attributed to impurities of methylacetylene or some of its deuterated derivatives.

The spectra were recorded with a 25-100 Jarrell Ash double monochromator by using a $90^{\circ}$ single pass configuration. The relative cross sections of the second order bands were measured with respect to an appropriate fundamental of the same sample and were then scaled by using the absolute values of Refs. 10 and 11. Exciting laser power of up to 9 $\mathrm{W}$ at $488 \mathrm{~nm}$ was necessary to measure the very weak $2 v_{1}$ and $2 v_{3}$ overtones of ${ }^{12} \mathrm{CH}_{4}$ and ${ }^{12} \mathrm{CD}_{4}$. The gas samples were contained in a cylindrical glass cell of low fluorescent material.

The experimental cross sections and wave numbers are reported in Table $I$, and the spectra of the $2 v_{1}, 2 v_{3}$, and $v_{3}+v_{4}$ bands of ${ }^{12} \mathrm{CH}_{4}$ are shown in Figs. 1 and 2 .

\section{THEORETICAL BACKGROUND}

In order to establish a relationship between the differential Raman scattering cross sections and the cubic force constants several intermediate steps are necessary.

In present work, the experimental cross sections related 
TABLE I. Experimental and calculated differential cross sections, $(\partial \sigma / \partial \Omega)$, for the mean polarizability Raman scattering of vibrational transitions in methane species. $(\partial \sigma / \partial \Omega)$ at $488 \mathrm{~nm}$ and $300 \mathrm{~K}$, in units of $10^{-39}\left[\mathrm{~m}^{2} \mathrm{sr}^{-1}\right]$.

\begin{tabular}{|c|c|c|c|c|c|c|}
\hline & & \multirow[b]{2}{*}{$v\left[\mathrm{~cm}^{-1}\right]$} & \multirow[b]{2}{*}{ Experimental } & \multicolumn{3}{|c|}{ Calculated } \\
\hline & & & & (a) & (b) & (c) \\
\hline & & 5769 & & & & \\
\hline \multirow[t]{8}{*}{${ }^{12} \mathrm{CH}_{4}$} & $2 v_{1}\left(A_{1}\right)$ & 5790 & $54 \pm 10$ & 53 & 58 & 231 \\
\hline & & 5804 & & & & \\
\hline & $2 v_{2}\left(A_{1}\right)$ & 3066 & $11000 \pm 3000^{e}$ & $\left\{\begin{array}{l}9420 \\
9467(P)\end{array}\right.$ & $\left\{\begin{array}{l}9420 \\
9467(P)\end{array}\right.$ & 1746 \\
\hline & $2 v_{3}\left(A_{1}\right)$ & $\left\{\begin{array}{l}5945 \\
\end{array}\right.$ & $81 \pm 16$ & t & ) & 61 \\
\hline & & 13909 & & $\int 9252$ & $\int 9225$ & \\
\hline & $2 v_{4}\left(A_{1}\right)$ & 2587 & $3900 \pm 800$ & $4983(P)$ & $\left\{\begin{array}{l}4989(P) \\
\text { ( }\end{array}\right.$ & 5810 \\
\hline & $v_{3}+v_{4}\left(A_{1}\right)$ & $\left\{\begin{array}{l}4314 \\
4342\end{array}\right.$ & $180 \pm 90$ & 117 & 126 & 71 \\
\hline & $v_{1}\left(A_{1}\right)$ & 2917 & $439000 \pm 24000$ & & & \\
\hline \multirow{8}{*}{${ }^{12} \mathrm{CD}_{4}$} & $2 \times(4)$ & 4153 & $(15-60)^{d}$ & 40 & 44 & 176 \\
\hline & $\left\langle V_{1}\left(A_{1}\right)\right.$ & l4188 & $(15-60)$ & $(5-750)$ & 44 & 170 \\
\hline & $2 v_{2}\left(A_{1}\right)$ & 2184 & $7160 \pm 2000^{e}$ & $\left\{\begin{array}{l}5750 \\
0\end{array}\right.$ & $\left\{\begin{array}{l}5750 \\
0\end{array}\right.$ & 1069 \\
\hline & $2 v,(A)$ & 4455 & $15-601$ & 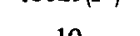 & 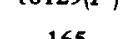 & 201 \\
\hline & $\left.2 v_{3}\left|\Omega_{1}\right|\right)$ & l4486 & $(13-00)$ & 10 & 105 & 381 \\
\hline & $2 v_{4}\left(A_{1}\right)$ & 1964 & $27000 \pm 5000^{e}$ & 101300 & 102120 & 3724 \\
\hline & $v_{3}+v_{4}\left(A_{1}\right)$ & 3877 & $920+230$ & $\begin{array}{l}127080(F) \\
425\end{array}$ & $\begin{array}{c}127900(P) \\
407\end{array}$ & 150 \\
\hline & $v_{1}\left(A_{1}\right)$ & 2108 & $313000 \pm 16000$ & & & \\
\hline \multirow{6}{*}{${ }^{12} \mathrm{CH}_{3} \mathrm{D}$} & & 2316 & & 2608 & 2608 & 3270 \\
\hline & $2 v_{4 b}\left(A_{1}\right)$ & 2316 & $20800 \pm 4000^{\circ}$ & $15800(P)$ & $15800(P)$ & $52 \%$ \\
\hline & $2 v_{2}\left(A_{1}\right)$ & 2910 & $205000 \pm 40000^{e}$ & $213000(P)$ & $213000(P)$ & 2020 \\
\hline & $2 v_{4 a}\left(A_{1}\right)$ & 2596 & $2800 \pm 500^{c}$ & 2870 & 2870 & 1900 \\
\hline & $v_{3 a}\left(A_{1}\right)$ & 2967 & $326000 \pm 33000$ & & & \\
\hline & $\nu_{1}\left(A_{1}\right\}$ & 2200 & $148000 \pm 8000$ & & & \\
\hline \multirow[t]{2}{*}{${ }^{12} \mathrm{CH}_{2} \mathrm{D}_{2}$} & $2 v_{2 a}\left(A_{1}\right)$ & 2860 & $9500 \pm 2000^{e}$ & $\left\{\begin{array}{c}8970 \\
0\end{array}\right.$ & 8970 & 1295 \\
\hline & $v_{3 a}\left(A_{1}\right)$ & 2975 & $237000 \pm 48000$ & & & \\
\hline
\end{tabular}

${ }^{a}$ With cubic field $(\mathrm{a})$ of Table III and $\left(2 \alpha^{\prime \prime}+\gamma^{\prime \prime}\right)_{\mathrm{CH}}=(4.81 \pm 1.0) \times 10^{-20}\left[\mathrm{C} \mathrm{V}^{-1}\right]$.

${ }^{b}$ With cubic force field (b) of Table III and $\left(2 \alpha^{n}+\gamma^{\prime \prime}\right)_{\mathrm{CH}}=(8.0 \pm 1.2) \times 10^{-20}\left[\mathrm{C} \mathrm{V}^{-1}\right]$.

' Harmonic approximation.

${ }^{\mathrm{d}}$ Lower and upper limits.

'Reference 11.

$(P)$ : Values calculated with the theory of perturbations [Eqs. (4) and (5)]. Otherwise results are from the contact transformation method [Eqs. (2) and (3)].

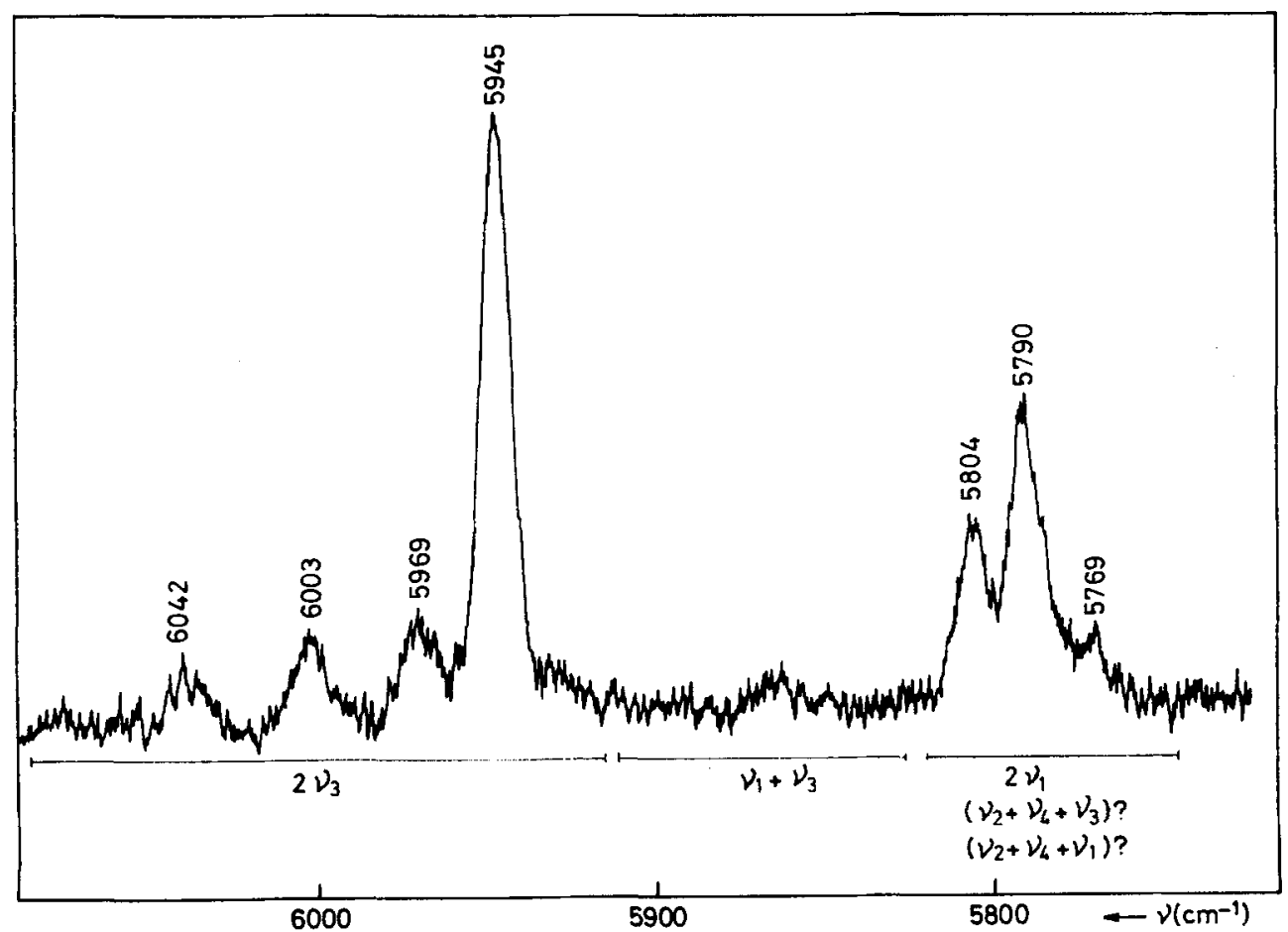

FIG. 1. Gas phase Raman spectrum of stretching overtones of ${ }^{12} \mathrm{CH}_{4}$. Cross section proportional to $45 \bar{\alpha}^{2}+7 \gamma^{2}$ ino analyzer); pressure 2 atm; laser power $\sim 8 \mathrm{~W}$; exciting line $=488 \mathrm{~nm}$. Peaks at $5969,5945,5804$, and 5790 $\mathrm{cm}^{-1}$ are strongly polarized. Peaks at 6003 and $6042 \mathrm{~cm}^{-1}$ are depolarized. 


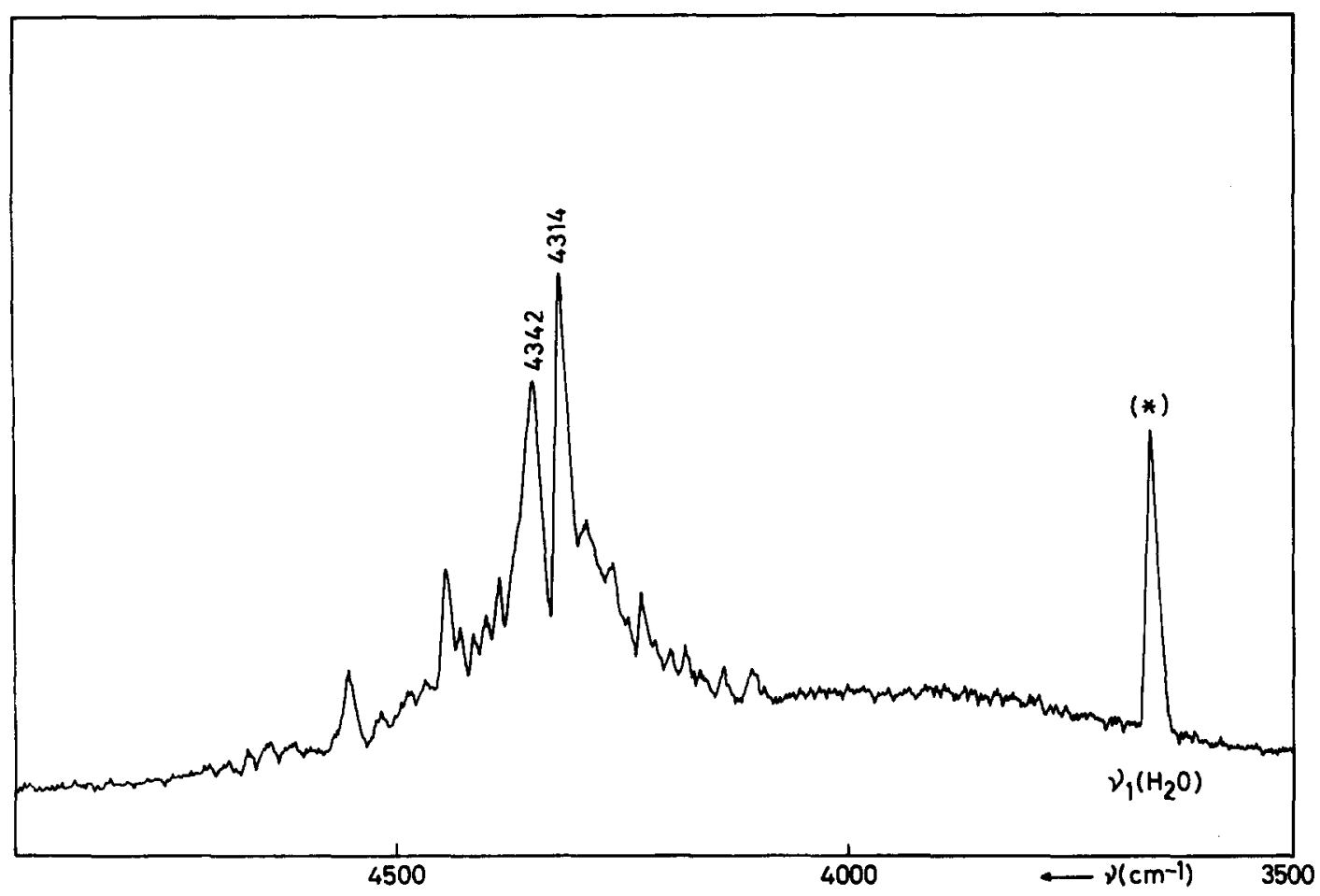

FIG. 2. Gas phase Raman spectrum of ${ }^{12} \mathrm{CH}_{4}$ in the region of $v_{3}+v_{4}$. Experimental conditions as in Fig. 1. $\left({ }^{*}\right) \mathrm{H}_{2} \mathrm{O}$ impurity of the ${ }^{12} \mathrm{CH}_{4}$ sample. The broad background centered at about $4000 \mathrm{~cm}^{-1}$ is due to the fluorescence of the cell.

to the mean polarizability scattering were used, since they usually appear as sharp bands not obscured by overlapping of the rovibrational structures.

In the SI system, ${ }^{12}$ the cross section for the $i \rightarrow j$ vibrational transition is

$$
\begin{aligned}
(\partial \sigma / \partial \Omega)_{i \rightarrow j}^{\text {mean pol. }}= & =\left(\pi / \epsilon_{0}\right)^{2}\left(v_{0}-v_{i j}\right)^{4}\langle i|\bar{\alpha}| j\rangle^{2}\left[Q_{\text {vib }}(T)\right]^{-1} \\
& \times \exp \left(-E_{i} / k T\right),
\end{aligned}
$$

where $\epsilon_{0}=8.8542 \times 10^{-12}\left[\mathrm{C} \mathrm{V}^{-1} \mathrm{~m}^{-1}\right]$ is the permittivity of vacuum and $v_{0}$ and $v_{i j}$ the wave numbers of the exciting radiation and of the Raman shift, respectively. $Q_{\text {vib }}(T)$ is the vibrational partition function of the molecule at the temperature $T$ of the sample. $|i\rangle$ and $\langle j\rangle$ are the vibrational wave functions for the initial $(i)$ and final $(j)$ vibrational states of the transition, and $\bar{\alpha}$ is the mean molecular polarizability operator. For methane species only the transition $0 \rightarrow j$ give an appreciable contribution to the vibrational scattering cross section at room temperature.

For practical reasons, the relevant transition moments of the type $\langle 0|\bar{\alpha}| j\rangle$ have been classified here in two groups:

\section{Weak vibrational resonances}

In these cases, the final level of an overtone or combination transition $j$ is far from any fundamental level of the molecule and the observed cross section, $(\partial \sigma / \partial \Omega)_{\mid \rightarrow \rightarrow j}^{\operatorname{mean} \text { pol. }}$, is usually below about $15 \%$ of the cross section of the closest fundamental bands.

Under these conditions the contact transformation of the polarizability operator ${ }^{13}$ yields a good convergence and the transition moment of the mean polarizability corresponding to an overtone $2 v_{l}$ is given by

$$
\begin{aligned}
\langle 0|\bar{\alpha}| j\rangle_{2 v_{l}}= & \frac{1}{2 \sqrt{2}}\left[\left(\frac{\partial^{2} \bar{\alpha}}{\partial q_{l}^{2}}\right)_{0}+\frac{2 k_{l l}}{\omega_{l}}\left(\frac{\partial \bar{\alpha}}{\partial q_{l}}\right)_{0}\right. \\
& \left.+\sum_{m \neq l} \frac{2 k_{l l m} \omega_{m}}{\left(4 \omega_{l}^{2}-\omega_{m}^{2}\right)}\left(\frac{\partial \bar{\alpha}}{\partial q_{m}}\right)_{0}\right]
\end{aligned}
$$

while for a combination $v_{l}+v_{l}$, one obtains

$$
\begin{aligned}
\langle 0|\bar{\alpha}| j\rangle_{v_{l}+v_{l^{\prime}}=} & \frac{1}{2}\left[\left(\frac{\partial^{2} \bar{\alpha}}{\partial q_{l} \partial q_{l^{\prime}}}\right)_{0}+\frac{2 k_{l l^{\prime}} \omega_{l}}{\omega_{l^{\prime}}\left(2 \omega_{l}+\omega_{l^{\prime}}\right)}\left(\frac{\partial \bar{\alpha}}{\partial q_{l}}\right)_{0}\right. \\
& +\frac{2 k_{l^{\prime} l^{\prime} l}}{\omega_{l}\left(2 \omega_{l^{\prime}}+\omega_{l}\right)}\left(\frac{\partial \bar{\alpha}}{\partial q_{l^{\prime}}}\right)_{0} \\
& \left.+\sum_{m \neq l, l^{\prime}} \frac{k_{l^{\prime} m} \omega_{m}}{\left[\left(\omega_{l}+\omega_{l^{\prime}}\right)^{2}-\omega_{m}^{2}\right]}\left(\frac{\partial \bar{\alpha}}{\partial q_{m}}\right)_{0}\right] \cdot(3)
\end{aligned}
$$

Here, $\partial \bar{\alpha} / \partial q_{l}$ and $\partial^{2} \bar{\alpha} / \partial q_{l} \partial q_{l}$, are derivatives of the mean molecular polarizability with respect to dimensionless normal coordinates $q ; \omega_{l}$ are the harmonic wave numbers and $k_{i j k}$ the cubic force constant in the representation of the coordinates $q$.

Provided the cubic constants $k_{l l m}$ or $k_{l l^{\prime} m}$ are not vanishing by symmetry, the resonant terms $\left(4 \omega_{l}^{2}-\omega_{m}^{2}\right)^{-1}$ and $\left[\left(\omega_{l}+\omega_{l}\right)^{2}-\omega_{m}^{2}\right]^{-1}$ in Eqs. (2) and (3) clearly show that $2 v_{l}$ and $v_{l}+v_{l}$, always are in vibrational resonance with the Raman active fundamentals $v_{m}$. These weak resonances have remarkable effects on the observed cross sections of second order transitions. Even in those cases where the difference $2 v_{l}-v_{m}$ or $v_{l}+v_{l},-v_{m}$ is larger than $1000 \mathrm{~cm}^{-1}$, the observed cross sections may change by more than one order of magnitude with respect to the unperturbed value. ${ }^{14}$ 


\section{Strong Fermi resonances}

If the final level of an overtone transition $2 v_{l}$ is close to a fundamental level of appropriate symmetry, its observed cross section may be larger than $15 \%$ of that of the fundamental band. For such a strong resonance Eq. (2) is no longer accurate because of the singularity $v_{m}=2 \omega_{l}$. Fortunately, only the interaction between these two levels needs to be taken into account in this case and the following expressions, derived from an exact diagonalization in the frame of the theory of perturbations ${ }^{15}$ may be safely used:

$$
\begin{aligned}
\langle 0|\bar{\alpha}| u\rangle= & (1 / 2)\left[\left(\frac{\partial \bar{\alpha}}{\partial q_{m}}\right)_{0} \sqrt{1+t}\right. \\
& \left. \pm(\sqrt{g} / 2)\left(\frac{\partial^{2} \bar{\alpha}}{\partial q_{l}^{2}}\right)_{0} \sqrt{1-t}\right], \\
\langle 0|\bar{\alpha}| l\rangle= & (1 / 2)\left[\left(\frac{\partial \bar{\alpha}}{\partial q_{m}}\right)_{0} \sqrt{1-t}\right. \\
& \left.\mp(\sqrt{g} / 2)\left(\frac{\partial^{2} \bar{\alpha}}{\partial q_{l}^{2}}\right)_{0} \sqrt{1+t}\right] .
\end{aligned}
$$

Here, $u$ and $l$ are the two final levels of the transition at upper and lower wave numbers, respectively; $g=1,2$, or 3 is the degeneracy of mode $q_{l}$, and $t$ is defined by the expression

$$
t= \pm\left[1-g\left(\frac{k_{l l m}}{X}\right)^{2}\right]^{1 / 2},
$$

where $|X|$ is the observed splitting of the Fermi diad. The upper signs set $( \pm)$ in Eqs. (4) and (5) stand for the case $k_{l m m}>0$, and the lower ( $\mp$ ), for $k_{l l m}<0$. The factor $t$ must be taken positive if $\omega_{m}^{*}-2 \omega_{l}^{*}>0$, and negative if $\omega_{m}^{*}$ $-2 \omega_{l}^{*}<0 ; \omega_{m}^{*}$ and $\omega_{l}^{*}$ are the "unperturbed" wave numbers, i.e., the harmonic wave numbers corrected by the anharmonicity constants $x_{s s^{\prime}}$ according to the expression

$$
\Delta E=\sum_{s<s^{\prime}} x_{s^{\prime}}\left(\mathrm{v}_{s}+\frac{g_{s}}{2}\right) \cdot\left(\mathrm{v}_{s^{\prime}}+\frac{g_{s^{\prime}}}{2}\right)
$$

for the energy increment of the unperturbed levels. For tetrahedral methane species the derivatives $\partial \bar{\alpha} / \partial q_{l}$ and $\partial^{2} \bar{\alpha} / \partial q_{l} \partial q_{l}$. in Eqs. (2) to (5) have been deduced from Table IV of Ref. 13 by using the transformation from mass weighted coordinates $Q$ to dimensionless coordinates $q$,

$$
\begin{aligned}
& \left(\partial \alpha / \partial q_{l}\right)=\sqrt{ } 2 b_{l}\left(\partial \alpha / \partial Q_{l}\right), \\
& \left(\partial^{2} \alpha / \partial q_{l} \partial q_{l^{\prime}}\right)=2 b_{l} b_{l^{\prime}}\left(\partial^{2} \alpha / \partial Q_{l}^{2}\right) .
\end{aligned}
$$

$b_{l}=\left(h / 8 \pi^{2} \omega_{l} c\right)^{1 / 2}$ is the zero vibrational amplitude. For nontetrahedral species the corresponding derivatives were calculated with the FORTRAN program OVER by using the same set of bond polarizability parameters. ${ }^{20}$

It should be noticed that, in the limit of negligible anharmonic interactions, i.e., when all $k_{i j k}$ cubic constants are negligibly small, Eq. (2) and Eqs. (4) or (5) are strictly equivalent. However, for finite values of the $k_{i j k}$ cubic constants, the results may be different, even for weak resonances. This is expected to be so, since Eq. (2) includes the interactions with all vibrational levels and Eqs. (4) and (5) only include the interaction with the closest vibrational level. Furthermore, Eq. (2) originates from the first two terms of the contact transformed polarizability in the expansion

$$
\begin{aligned}
\alpha_{\mathrm{eff}}= & \alpha+i[S, \alpha]-\frac{1}{2}[S,[S, \alpha]] \\
& -(i / 6)[S,[S,[S, \alpha]]]+\cdots,
\end{aligned}
$$

where the contribution of the neglected terms $\frac{1}{2}[S,[S, \alpha]]+(i / 6)[S,[S,[S, \alpha]]]$ is expected to be small, while Eqs. (4) and (5) arise from the exact diagonalization of a $2 \times 2$ perturbation Hamiltonian. ${ }^{15}$ The numerical results from both methods (a) and (b) are discussed in more detail in the final part of this work.

\section{NUMERICAL CALCULATIONS}

According to previous expressions and to Ref. 13, the observed Raman scattering cross sections of second order bands provide information about the quadratic and cubic terms of the force field and about the equilibrium and the first and second derivatives of the $\mathrm{CH}$ bond polarizability tensor.

For calculating the scattering cross section due to the mean polarizability of methane, only the bond parameters $\left(2 \alpha^{\prime}+\gamma^{\prime}\right)_{\mathrm{CH}}$ and $\left(2 \alpha^{\prime \prime}+\gamma^{\prime \prime}\right)_{\mathrm{CH}}$ are necessary. The parameter ${ }^{10}$

$$
\left(2 \alpha^{\prime}+\gamma^{\prime}\right)_{\mathrm{CH}}=3.787 \times 10^{-30}\left[\mathrm{C} \mathrm{V}^{-1} \mathrm{~m}\right],
$$

and the quadratic force field ${ }^{7}$ have been taken from the literature and are supposed to be quite accurately determined. Its refinement has not been attempted here. The parameter $\left(2 \alpha^{\prime \prime}+\gamma^{\prime \prime}\right)_{\mathrm{CH}}$ has been determined and 10 cubic force constants in symmetry coordinates have been refined by using experimental cross section data of Ref. 11 and of present work, according to the following procedure:

From Eq. (1), the square of the transition moment $\langle 0|\bar{\alpha}| j\rangle$ can be derived from the experimental cross section of the corresponding Raman band. By substituting the two possible experimental values of the transition moment (differing in sign) in Eqs. (2), (3), (4), or (5), it was possible to derive either two possible "experimental" values for each $k_{r s t}$ [Eqs. (4), (5), and (6)], or two possible relations between the various experimental $k_{r s t}$ 's appearing in Eqs. (2) or (3). From these values and relations, and by using the transformation

$$
\begin{aligned}
k_{r s t}= & \frac{2 \sqrt{2} b_{r} b_{s} b_{t}}{\left(1+\delta_{r s}\right)\left(1+\delta_{r t}+\delta_{s t}\right)} \\
& \times\left[F_{i j k} L_{i}^{r} L_{j}^{s} L_{k}^{t}+F_{i j}\left(L_{i}^{r s} L_{j}^{t}+L_{i}^{r t} L_{j}^{s}+L_{i}^{s t} L_{j}^{r}\right)\right],
\end{aligned}
$$

it was possible to express the experimental information as a system of 14 linear equations with 10 symmetry force constants $F_{111}, F_{122}, F_{133}, F_{134}, F_{144}, F_{234}, F_{244}, F_{334}, F_{344}$, and $F_{444}$, and the parameter $\left(2 \alpha^{\prime \prime}+\gamma^{\prime \prime}\right)_{\mathrm{CH}}$ as unknowns. Due to the uncertainty in the sign of the transition moments the system had a multiplicity of numerical solutions.

On the other hand, the structure of the system was such that the constants $F_{111}, F_{122}, F_{133}, F_{134}, F_{144}$, and the parameter $2 \alpha^{\prime \prime}+\gamma^{\prime \prime}$ were overdetermined, but only four constants among $F_{234}, F_{244}, F_{344}, F_{444}$, and $F_{334}$ could be simultaneously determined from the available experimental data. In order to get a value for these five constants, the condition of minimal deviation from the previously reported values ${ }^{2-7}$ was 
explicitly imposed. Under these conditions only two satisfactory solutions were found. They are reported in Table III.

It is worthwhile to point out that imposing external constraints was necessary just as a consequence of the reduced number of experimental data used in present work, but is not a concomitant limitation of the proposed method. In fact, over 200 significant intensity data are in principle observable, and should be used in the future refinement of the cubic force field of methane.

\section{DISCUSSION}

It is interesting to note that most of the refined $F_{i j k}$ 's of Table III appear to be well determined, despite the relatively low accuracy of the experimental cross sections. This is due to the high sensitivity of the transition moments [Eqs. (2)-(5)] vs the cubic constants $k_{r s t}$. This effect is enhanced by the fact that the scattering cross sections depend on the square of the transition moments, and so, variations of a few percent in the cubic force constants may induce changes in some of the calculated cross sections of more than one order of magnitude. However, it should be emphasized that the uncertainties quoted for $F_{i j k}$ 's in Table III are not necessarily the actual uncertainty of the physical magnitude, since the method here used is based on the model of bond polarizabilities. We believe that the derivatives $\left(\partial \bar{\alpha} / \partial q_{l}\right)_{0}$ and $\left(\partial^{2} \bar{\alpha} / \partial q_{l} \partial q_{l}\right)_{0}$ on
Table II, which were calculated with the bond polarizability model, are accurate to nearly $10 \%$ but systematic deviations larger than this value cannot be excluded, at present. If this is the case, systematic errors in some of the $\left(\partial \bar{\alpha} / \partial q_{l} \partial q_{l^{\prime}}\right)_{0}$ should propagate in the numerical calculation yielding not too accurate values for the $F_{i j k}$ 's but, probably, mantaining a good consistency for the overall system. Since the values here used for $\left(\partial^{2} \bar{\alpha} / \partial q_{l} \partial q_{l}^{\prime}\right)_{0}$ cannot be directly checked, for instance, by comparing them with ab initio results, the only way to estimate the accuracy of the $F_{i j k}$ 's is either to compare them with values reported by other authors using a different methodology or by calculating spectroscopic constants that are known on the basis of the observed wave numbers of rovibrational bands.

Our two possible solutions (a) and (b) in Table III may be compared with $F_{i j k}$ 's from Refs. 2-7. For solution (a), corresponding to the bond parameter $\left(2 \alpha^{\prime \prime}+\gamma^{\prime \prime}\right)_{\mathrm{CH}}$ $=(4.81 \pm 1.0) \times 10^{-20}\left[\mathrm{C} \mathrm{V}^{-1}\right]$, the constants $F_{111}, F_{122}$, $F_{133}, F_{244}$, and $F_{444}$ agree well with the results reported by Gray and Robiette, ${ }^{7}$ but, for $F_{134}$ and $F_{144}$ our results are closer to those of the early work of Kuchitsu and Bartell. ${ }^{2}$ For the remaining constants, the literature $F_{i j k}$ values reported on Table III are so contradictory that no further conclusions about the quality of our results can be reached in

TABLE II. Derivatives $\left(D^{n)}=\partial \bar{\alpha} / \partial q_{i}\right.$ or $\left.\partial^{2} \bar{\alpha} / \partial q_{i} \partial q_{j}\right)$ of the mean polarizability of $T_{d}$ symmetry methane species with respect to the dimensionless normal coordinates of the harmonic force field of Ref. $7 . D^{n)}$ in units of $10^{-40}\left[\mathrm{C} \mathrm{V}^{-1} \mathrm{~m}^{2}\right] ; \omega$ 's are calculated wave numbers.

\begin{tabular}{|c|c|c|c|c|c|c|c|c|c|c|c|c|}
\hline & \multicolumn{3}{|c|}{${ }^{12} \mathrm{CH}_{4}$} & \multicolumn{3}{|c|}{${ }^{13} \mathrm{CH}_{4}$} & \multicolumn{3}{|c|}{${ }^{12} \mathrm{CD}_{4}$} & \multicolumn{3}{|c|}{${ }^{13} \mathrm{CD}_{4}$} \\
\hline & $\omega_{i}$ & $\omega_{j}$ & $D^{n !}$ & $\omega_{i}$ & $\omega_{j}$ & $D^{n)}$ & $\omega_{i}$ & $\omega_{j}$ & $D^{n)}$ & $\omega_{i}$ & $\omega_{j}$ & $D^{n j}$ \\
\hline$\partial \bar{\alpha} / \partial q_{1}$ & 3025 & $\cdots$ & 0.2655 & 3025 & ... & 0.2655 & 2140 & $\ldots$ & 0.2233 & 2140 & $\cdots$ & 0.2233 \\
\hline$\partial^{2} \bar{\alpha} / \partial q_{1}^{2}$ & 3025 & 3025 & 0.0177 & 3025 & 3025 & 0.0177 & 2140 & 2140 & 0.0125 & 2140 & 2140 & 0.0125 \\
\hline$\partial^{2} \bar{\alpha} / \partial q_{2 \sigma}^{2}(\sigma=a, b)$ & 1583 & 1583 & 0.0246 & 1583 & 1583 & 0.0246 & 1120 & 1120 & 0.0174 & 1120 & 1120 & 0.0174 \\
\hline$\partial^{2} \bar{\alpha} / \partial q_{3 \sigma}^{2}(\sigma=x, y, z)$ & 3157 & 3157 & 0.0190 & 3146 & 3146 & 0.0189 & 2336 & 2336 & 0.0147 & 2320 & 2320 & 0.0145 \\
\hline$\partial^{2} \bar{\alpha} / \partial q_{4 \sigma}^{2}(\sigma=x, y, z)$ & 1367 & 1367 & 0.0345 & 1359 & 1359 & 0.0343 & 1034 & 1034 & 0.0259 & 1025 & 1025 & 0.0257 \\
\hline$\partial^{2} \bar{\alpha} / \partial q_{3 \sigma} \partial q_{4 \sigma}(\sigma=x, y, z)$ & 3157 & 1367 & 0.0033 & 3146 & 1359 & 0.0031 & 2336 & 1034 & 0.0042 & 2320 & 1025 & 0.0040 \\
\hline
\end{tabular}

TABLE III. Cubic force constants of methane $F_{i j k}$ in the representation of symmetry coordinates of Ref. 7.

\begin{tabular}{|c|c|c|c|c|c|c|c|}
\hline & \multicolumn{2}{|c|}{ This work } & \multirow[b]{2}{*}{$\begin{array}{c}\text { Gray and } \\
\text { Robiette (Ref. 7) }\end{array}$} & \multirow[b]{2}{*}{$\begin{array}{l}\text { Hirota } \\
\text { (Ref. 6) }\end{array}$} & \multirow[b]{2}{*}{$\begin{array}{c}\text { Pulay, Mayer } \\
\text { and Boggs (Ref. 4) }\end{array}$} & \multirow[b]{2}{*}{$\begin{array}{c}\text { Bartell and } \\
\text { Kuchitsu (Ref. 2) }\end{array}$} & \multirow[b]{2}{*}{$\begin{array}{l}\text { Kuchitsu and } \\
\text { Bartell (Ref. 3) }\end{array}$} \\
\hline & (a) & (b) & & & & & \\
\hline$F_{111}{ }^{\mathrm{c}}$ & $-15.32 \pm 2.08$ & $-12.06 \pm 2.54$ & -15.30 & -12.40 & -15.08 & -17.21 & -19.30 \\
\hline$F_{122}^{c}$ & $-0.322 \pm 0.020$ & $-0.322 \pm 0.022$ & -0.299 & -0.33 & -0.227 & -0.193 & -0.466 \\
\hline$F_{133}{ }^{c}$ & $-15.58 \pm 2.48$ & $-15.80 \pm 2.74$ & -15.69 & -14.08 & -15.47 & -14.86 & -15.74 \\
\hline$F_{134}{ }^{d}$ & $0.347 \pm 0.190$ & $0.292 \pm 0.200$ & & 0.25 & 0.066 & 0.180 & 0.637 \\
\hline$F_{144}$ & $-0.371 \pm 0.020$ & $-0.368 \pm 0.022$ & -0.110 & 0.14 & -0.196 & -0.195 & -0.466 \\
\hline$F_{222}{ }^{\mathrm{f}}$ & & & 0.094 & 0.058 & 0.098 & & \\
\hline$F_{233}{ }^{d}$ & & & & -0.22 & -0.370 & & \\
\hline$F_{234}{ }^{e}$ & $0.258 \pm 0.055$ & $0.258 \pm 0.063$ & & 0.29 & 0.160 & & \\
\hline$F_{244}{ }^{f}$ & $-0.319 \pm 0.167$ & $-0.319 \pm 0.184$ & -0.311 & -0.37 & -0.386 & & \\
\hline$F_{333}{ }^{c}$ & & & -15.87 & -13.48 & -15.65 & -13.83 & -13.96 \\
\hline$F_{334}^{d}$ & $-0.293 \pm 0.755$ & $-0.293 \pm 0.831$ & & 0.18 & -0.268 & & -10.70 \\
\hline$F_{344^{e}}$ & $0.045 \pm 0.077$ & $0.045 \pm 0.083$ & & -0.77 & -0.101 & & \\
\hline$F_{444}{ }^{f}$ & $0.328 \pm 0.245$ & $0.328 \pm 0.276$ & 0.345 & 0.233 & 0.426 & & \\
\hline
\end{tabular}

- For $\left(2 a^{\prime \prime}+\gamma^{\prime \prime}\right)_{\mathrm{CH}}=(4.81 \pm 1.00) \times 10^{-20}\left[\mathrm{C} \mathrm{V}^{-1}\right]$; (preferred solution).

${ }^{b}$ For $\left(2 \alpha^{\prime \prime}+\gamma^{\prime \prime}\right)_{\mathrm{CH}}=(8.00 \pm 1.20) \times 10^{-20}\left[\mathrm{C} \mathrm{V}^{-1}\right]$; (alternative solution).

caJ $\AA^{-3}$

aJ $\AA^{-2}$

e aJ $\AA^{-1}$

f aJ 
TABLE IV. Cubic force constants for $T_{d}$ symmetry methane species, $k_{r s}$, in the representation of dimensionless normal coordinates, corresponding to solution (a) of Table III. $k_{r s t}$ in $\mathrm{cm}^{-1}$.

\begin{tabular}{|c|c|c|c|c|c|}
\hline & \multicolumn{3}{|c|}{ This work } & \multicolumn{2}{|c|}{$\begin{array}{l}\text { Gray and Robiette } \\
\text { (Ref. 7) }\end{array}$} \\
\hline & ${ }^{12} \mathrm{CH}_{4}$ & ${ }^{12} \mathrm{CD}_{4}$ & ${ }^{13} \mathrm{CH}_{4}$ & ${ }^{12} \mathrm{CH}_{4}$ & ${ }^{12} \mathrm{CD}_{4}$ \\
\hline$k_{111}$ & -149.23 & -88.77 & -149.20 & -149.33 & -88.83 \\
\hline$k_{122}$ & 17.52 & 10.42 & 17.52 & 21.00 & 12.50 \\
\hline$k_{133}$ & -480.40 & -296.80 & -478.80 & -487.00 & -302.50 \\
\hline$k_{134}$ & 13.18 & 41.19 & 8.68 & -19.00 & 25.00 \\
\hline$k_{144}$ & 81.44 & 50.82 & 80.97 & 114.50 & 72.50 \\
\hline$k_{222}$ & -8.87 & -5.28 & -8.87 & -8.83 & -5.33 \\
\hline$k_{233}$ & -44.20 & -34.21 & -43.14 & -46.00 & -37.00 \\
\hline$k_{234}$ & -119.17 & -74.41 & -118.59 & -147.00 & -92.00 \\
\hline$k_{244}$ & -18.84 & -9.02 & -19.13 & -18.00 & -8.00 \\
\hline$k_{333}$ & -171.62 & -112.54 & -170.25 & -171.83 & -112.83 \\
\hline$k_{334}$ & -61.84 & -33.23 & -62.29 & -63.00 & -36.00 \\
\hline$k_{344}$ & -56.61 & -37.55 & -56.09 & -76.50 & -50.00 \\
\hline$k_{444}$ & -21.61 & -12.73 & -21.60 & -21.17 & -12.00 \\
\hline
\end{tabular}

this way. Solution (b), for $\left(2 \alpha^{\prime \prime}+\gamma^{\prime \prime}\right)_{\mathrm{CH}}=(8.0 \pm 1.2)$ $\times 10^{-20}\left[\mathrm{C} \mathrm{V}^{-1}\right]$, yields slightly larger uncertainties. Consequently, we shall provisionally prefer solution (a).

The cubic constants $k_{r s t}$ in the representation of dimensionless normal coordinates corresponding to solution (a) are reported in Table IV for some tetrahedral methane species. They have been calculated with Eq. (11), and the constants $F_{222}, F_{233}$, and $F_{333}$, not refined in present work, were kept fixed to the $a b$ initio values of Pulay, Mayer, and Boggs. ${ }^{4}$ The corresponding $k_{r s t}$ constants, reported by Gray and Robiette, ${ }^{7}$ are reproduced in the same table, the only significant differences being localized on the constants $k_{134}, k_{144}, k_{234}$, and $k_{344}$.
The $\hat{H}_{22}$ spectroscopic constants $Y_{1}, Y_{2}, Y_{3}, Y_{4}, Z_{3 s}$, $Z_{4 s}, Z_{3 t}, Z_{4 t}$, and $Z_{2}$ according to the nomenclature of Hecht, ${ }^{16}$ were calculated for the derivatives ${ }^{12} \mathrm{CH}_{4},{ }^{12} \mathrm{CD}_{4}$, ${ }^{13} \mathrm{CH}_{4}$, and ${ }^{13} \mathrm{CD}_{4}$ by using the $k_{r s t}$ force constants of Table IV. They are shown in Table V, together, with experimental results originating from different sources [Refs. 7, 8, and references therein]. Our $\hat{H}_{22}$ constants are not too different from those calculated by Gray and Robiette, ${ }^{7}$ due to the fact that they mainly depend on $k_{r s t}$ constants that are similar in both force fields. On the other hand, for some of the $\hat{H}_{22}$ constants, as for instance $Z_{3 t}$ in ${ }^{12} \mathrm{CH}_{4}$ and ${ }^{13} \mathrm{CH}_{4}$, the difference between theoretical and experimental results is quite large for any of the two independently determined cubic force fields. These discrepancies suggest that the use of $\hat{H}_{22}$ coefficients might not always be safe for determining the cubic force field.

We have also calculated the $F_{2}, F_{3 s}, F_{3 t}, F_{4 s}$, and $F_{4 t}$ coefficients by using the expressions derived by Aliev and Watson. ${ }^{17}$ Our results are nearly the same as those reported by Gray and Robiette, ${ }^{7}$ since the influence of those terms depending on $k_{134}, k_{144}, k_{234}$, and $k_{344}$ constants, which are different in both force fields, is quite small. Thus, the discrepancies between calculated and experimental $F$ coefficients that were pointed out by Gray and Robiette cannot be satisfactorily explained on the basis to the inaccuracy of the cubic $k_{r s t}$ constants. More likely, the $F$ coefficients depend on higher order vibration-rotation terms not included in the expressions of Aliev and Watson.

It has been already pointed out that weak Fermi diads might be alternatively described in two ways, with similar results. Among the spectra utilized in present work the following weak Fermi diads are present: $2 v_{2}: v_{1}$ in ${ }^{12} \mathrm{CH}_{4}$ and ${ }^{12} \mathrm{CD}_{4} ; 2 v_{4}: v_{1}$ in ${ }^{12} \mathrm{CH}_{4}$ and ${ }^{12} \mathrm{CD}_{4} ; 2 v_{4 b}: v_{1}$ in ${ }^{12} \mathrm{CH}_{3} \mathrm{D}$ and $2 v_{2 a}: v_{3 a}$ in ${ }^{12} \mathrm{CH}_{2} \mathrm{D}_{2}$. The cross sections calculated accord-

TABLE V. Calculated and experimental $\hat{H}_{22}$ coefficients for ${ }^{12} \mathrm{CH}_{4},{ }^{13} \mathrm{CH}_{4},{ }^{12} \mathrm{CD}_{4}$, and ${ }^{13} \mathrm{CD}_{4}\left(\right.$ in $\mathrm{cm}^{-1}$ ).

\begin{tabular}{|c|c|c|c|c|c|c|c|}
\hline \multirow[b]{2}{*}{ e } & \multicolumn{2}{|c|}{${ }^{12} \mathrm{CH}_{4}$} & \multicolumn{2}{|c|}{${ }^{13} \mathrm{CH}_{4}$} & \multicolumn{2}{|c|}{${ }^{12} \mathrm{CD}_{4}$} & \multirow{2}{*}{$\frac{{ }^{13} \mathrm{CD}_{4}}{\text { calc }^{\mathrm{a}}}$} \\
\hline & $\mathrm{calc}^{\mathrm{a}}$ & expt & calc $^{\mathrm{a}}$ & expt & $\mathrm{call}^{\mathrm{a}}$ & expt & \\
\hline$Y_{1}$ & -0.0382 & $-0.0377^{\mathrm{d}}$ & -0.0382 & & -0.0136 & & -0.0136 \\
\hline$Y_{2}$ & 0.0877 & $\left\{\begin{array}{l}0.0879^{b} \\
0.0910^{c}\end{array}\right.$ & 0.0822 & & 0.0717 & $0.0639^{b}$ & 0.0628 \\
\hline$Y_{3}$ & -0.0347 & $-0.0357^{\mathrm{d}}$ & -0.0342 & $\left\{\begin{array}{l}-0.0400^{\mathrm{b}} \\
-0.0399^{\mathrm{c}}\end{array}\right.$ & -0.0146 & $\left\{\begin{array}{l}-0.0132^{b} \\
-0.0134^{c}\end{array}\right.$ & -0.0142 \\
\hline$Y_{4}$ & -0.0682 & $\left\{\begin{array}{l}-0.0647^{b} \\
-0.0635^{c}\end{array}\right.$ & -0.0649 & $\left\{\begin{array}{l}-0.0598^{b} \\
-0.0598^{c}\end{array}\right.$ & -0.0498 & $-0.0543^{b}$ & -0.0441 \\
\hline$Z_{3 s}$ & -0.0053 & $\left\{\begin{array}{r}-0.0075^{b} \\
-0.0076^{c} \\
10.0138^{b}\end{array}\right.$ & -0.0047 & {$\left[\begin{array}{r}-0.0066^{\mathrm{b}} \\
-0.0067^{\mathrm{c}} \\
{\left[0.0159^{\mathrm{b}}\right.}\end{array}\right.$} & -0.0037 & $\left\{\begin{array}{l}-0.0036^{b} \\
-0.0038^{c}\end{array}\right.$ & -0.0030 \\
\hline$Z_{4 s}$ & 0.0133 & $\left\{\begin{array}{l}0.0138^{\circ} \\
0.0135^{c}\end{array}\right.$ & 0.0142 & $\left\{\begin{array}{l}0.0159^{\circ} \\
0.0158^{\mathrm{c}}\end{array}\right.$ & -0.0023 & $-0.0060^{\mathrm{b}}$ & -0.0006 \\
\hline$Z_{3 r}$ & -0.0019 & $\left\{\begin{array}{l}-0.0064^{\mathrm{o}} \\
-0.0031^{\mathrm{c}} \\
-0.0303^{\mathrm{b}}\end{array}\right.$ & -0.0017 & $\left\{\begin{array}{l}-0.0051^{\circ} \\
-0.0026^{c}\end{array}\right.$ & -0.0013 & $-0.0009^{b}$ & -0.0012 \\
\hline$Z_{4 t}$ & -0.0293 & $\left\{\begin{array}{l}-0.0303^{\mathrm{b}} \\
-0.0151^{\mathrm{c}}\end{array}\right.$ & -0.0277 & $\left\{\begin{array}{l}-0.0292^{\circ} \\
-0.0146^{c}\end{array}\right.$ & -0.0221 & $-0.0201^{b}$ & -0.0195 \\
\hline$z_{2}$ & -0.0102 & $0.0396^{\mathrm{c}}$ & -0.0138 & & 0.0341 & & 0.0282 \\
\hline
\end{tabular}

Calculated using the $k_{r s t}$ force constants of present work [solution (a)] and the expressions of Hecht (Ref. 16).

bexperimental values from different sources quoted in Ref. 7.

'Experimental values from different sources quoted in Ref. 8.

d Reference 19.

'Equivalence between Hecht's (Ref. 16) and Herranz's (Ref. 18) nomenclature: $Y_{i}=-\alpha_{i} ; i=1,2,3,4 ; Z_{j t}=-\delta_{j} / 10 ; Z_{j s}=3 \beta, j=3,4$. 
ing to both methods are shown in Table I. There, $P$ stands for the exact diagonalization. Otherwise results are from the contact transformation method. It may be seen that the results are similar only for $2 v_{2}: v_{1}$ in ${ }^{12} \mathrm{CH}_{4}$ and ${ }^{12} \mathrm{CD}_{4}$ and for $2 v_{2 a}: v_{3 a}$ in ${ }^{12} \mathrm{CH}_{2} \mathrm{D}_{2}$ but, for the other resonances, serious discrepancies are evident. This might be due to two independent factors: First, in the contact transformation method, the transition moments were defined from the first term of the contact transformed polarizability, namely $\alpha_{\text {eff }}$ $=\alpha_{0}+i[S, \alpha]$, neglecting the higher order terms; second, some of the harmonic wave numbers appearing in Eqs. (2) and (3), which were taken from Ref. 7 might still have considerable errors, perhaps larger than $10 \mathrm{~cm}^{-1}$. Such inaccuracy in the wave numbers may introduce appreciable errors in the cross sections of second order bands, especially in the case of vibrational resonances. Thus, the expressions derived from the exact diagonalization [Eqs. (4) and (5)], which appear as a function of the observed wave numbers rather than of the harmonic ones, were preferentially used in the computational refinement of the cubic force constants.

Finally, it is worthwhile to consider the scattering cross sections of second order transitions calculated in the harmonic approximation [Table I, row (c)]. It is evident that the harmonic approximation is almost useless for interpreting such spectroscopic features since deviations of more than one order of magnitude with respect to the experimental value arise commonly from this approximation.

\section{CONCLUSION}

In view of the results here presented, we believe that the use of second order vibrational Raman intensities represents an interesting approach towards the evaluation of the cubic force field of a molecule, provided the harmonic force field is well established.

In principle, this method yields very accurate cubic force constants, since the second order vibrational intensities are extremely sensitive to the anharmonicity. However, at present, this accuracy is limited by that of the second deriva- tives of the molecular polarizability with respect to the normal coordinates. Since the uncertainty of the second derivatives of the polarizability calculated according to the bond polarizability model is not accurately known the cubic force field of methane here reported should be considered only as a first step of a new methodology for studying the vibrational anharmonicity.

\section{ACKNOWLEDGMENTS}

We are indebted to J. Brandmüller and $\mathbf{H}$. W. Schrötter for moral support and to the Stiftung Volkswagenwerk of the Federal Republic of Germany for providing the high power laser source to obtain some of the results presented here.

'Z. Cihla and A. Chedin, J. Mol. Spectrosc. 40, 337 (1971)

${ }^{2}$ K. Kuchitsu and L. S. Bartell, J. Chem. Phys. 36, 2470 (1962).

${ }^{3}$ L. S. Bartell and K. Kuchitsu, J. Chem. Phys. 68, 1213 (1978).

${ }^{4}$ P. Pulay, W. Meyer, and J. E. Boggs, J. Chem. Phys. 68, 5077 (1978).

${ }^{5}$ P. Pulay, G. Fogarasi, I. Pang, and J. E. Boggs, J. Am. Chem. Soc. 101, 2550 (1979).

${ }^{6}$ E. Hirota, J. Mol. Spectrosc. 77, 213 (1979).

'D. L. Gray and G. A. Robiette, Mol. Phys. 37, 1901 (1979).

${ }^{8}$ Y. S. Makushkin, V. N. Savelev, and O. N. Ulenikov, Opt. Spectrosc. 48 264 (1980).

${ }^{9}$ W. F. Murphy and S. Montero, Mol. Phys. 44, 187 (1981).

${ }^{10}$ S. Montero and D. Bermejo, Mol. Phys. 32, 1229 (1976).

${ }^{11}$ D. Bermejo, R. Escribano, and J. M. Orza, J. Mol. Spectrosc. 65, 345 (1977).

${ }^{12}$ J. Martin and S. Montero, J. Chem. Phys. 80, 4610 (1984).

${ }^{13}$ S. Montero, J. Chem. Phys. 77, 23 (1982).

${ }^{14}$ C. Domingo, R. Escribano, W. F. Murphy, and S. Montero, J. Chem. Phys. 77, 4353 (1982).

${ }^{15}$ S. Montero, J. Chem. Phys. 79, 4091 (1983).

${ }^{16}$ K. T. Hecht, J. Mol. Spectrosc. 5, $355(1960)$.

${ }^{17}$ M. R. Aliev and J. K. G. Watson, J. Mol. Spectrosc. 75, 157 (1979).

${ }^{18}$ J. Herranz, J. Mol. Spectrosc. 6, 343 (1961).

${ }^{19} \mathrm{G}$. Poussigue, E. Pascaud, J. P. Champion, and G. Pierre, J. Mol. Spectrosc. 93, 351 (1982).

${ }^{20}$ The program OVER performs the numerical calculation of Eqs. (8), (9), and (10) of Ref. 13. 